\title{
Heritability estimates for Mycobacterium avium subspecies paratuberculosis status of German Holstein cows tested by fecal culture
}

\author{
J. Küpper, ${ }^{\star}$ H. Brandt, ${ }^{*}$ K. Donat, $†$ and G. Erhardt ${ }^{\star 1}$ \\ *Institut für Tierzucht und Haustiergenetik, Justus-Liebig-Universität, Ludwigstraße 21b, 35390 Gießen, Germany \\ †Thüringer Tierseuchenkasse, Victor-Goerttler-Str. 4, 07745 Jena, Germany
}

\begin{abstract}
The objective of this study was to estimate genetic manifestation of Mycobacterium avium ssp. paratuberculosis (MAP) infection in German Holstein cows. Incorporated into this study were 11,285 German Holstein herd book cows classified as MAP-positive and MAPnegative animals using fecal culture results and originating from 15 farms in Thuringia, Germany involved in a paratuberculosis voluntary control program from 2008 to 2009. The frequency of MAP-positive animals per farm ranged from 2.7 to $67.6 \%$. The fixed effects of farm and lactation number had a highly significant effect on MAP status. An increase in the frequency of positive animals from the first to the third lactation could be observed. Threshold animal and sire models with sire relationship were used as statistical models to estimate genetic parameters. Heritability estimates of fecal culture varied from 0.157 to 0.228 . To analyze the effect of prevalence on genetic parameter estimates, the total data set was divided into 2 subsets of data into farms with prevalence rates below $10 \%$ and those above $10 \%$. The data set with prevalence above $10 \%$ show higher heritability estimates in both models compared with the data set with prevalence below $10 \%$. For all data sets, the sire model shows higher heritabilities than the equivalent animal model. This study demonstrates that genetic variation exists in dairy cattle for paratuberculosis infection susceptibility and furthermore, leads to the conclusion that MAP detection by fecal culture shows a higher genetic background than ELISA test results. In conclusion, fecal culture seems to be a better trait to control the disease, as well as an appropriate feature for further genomic analyses to detect MAP-associated chromosome regions.
\end{abstract}

Key words: Mycobacterium avium ssp. paratuberculosis, heritability, fecal culture

Received September 29, 2011.

Accepted December 30, 2011.

${ }^{1}$ Corresponding author: Georg.Erhardt@agrar.uni-giessen.de

\section{INTRODUCTION}

Paratuberculosis (ParaTB) or Johne's disease (JD) is a widespread chronic enteritis of ruminants caused by the agent Mycobacterium avium ssp. paratuberculosis (MAP). The animals are infected as calves but due to the characteristically long incubation time of up to 15 yr, they initially show no clinical symptoms (Chiodini et al., 1984). The first period of illness is characterized by intense, intermittent, and chronic diarrhea, causing weight loss to the point of death (Chiodini et al., 1984). High economic losses are incurred through diminished milk and fattening performance, decreased fertility, as well as costs associated with the management of infected animals (McKenna et al., 2006).

Strategies decreasing the incidence of paratuberculosis so far involve the detection and culling of infected animals as well as other management strategies to avoid further dispersion of MAP. The diagnosis of MAP-infected animals can be carried out directly via fecal PCR, using fecal or tissue culture for agent detection, or indirectly through MAP antibody detection in blood or milk with commercial ELISA tests (Harris and Barletta, 2001). The problem with all of these tests is the limited sensitivity or specificity to detect infected animals at an early stage of infection (Nielsen and Toft, 2008). To improve the accuracy of diagnosis, it is possible and advisable to combine different detection methods (Böttcher and Gangl, 2004).

Due to long incubation times, subclinical phases (Chiodini et al., 1984) and problematic diagnostics, a definite statement on MAP occurrence is hardly possible. The highest MAP prevalence of single herds is based on extrapolations of single regions of the country (Dreier et al., 2006). For example, herd prevalence rates in Europe range from $6.9 \%$ in Austria (Dreier et al., 2006) to $54.7 \%$ in the Netherlands (Muskens et al., 2000). In the United States, prevalence was estimated to be $87.5 \%$ (Wells et al., 2002) and for Canada between 34.0 and $58.0 \%$ (Hendrick et al., 2005). Within-herd prevalences are found in a wide range from below $1 \%$ (Donat et al. 2011) to more than 30\% (Gasteiner et al., 1999; Hinger et al., 2008). 
Heritabilities for MAP status were first estimated in the Netherlands by Koets et al. (2000). Further studies estimated heritabilities between 0.031 and 0.159 (Mortensen et al., 2004; Gonda et al., 2006; Hinger et al., 2008; Attalla et al., 2010; van Hulzen et al., 2011). Differences in estimated heritability results could be caused by the various diagnostic methods used to detect MAP, divergent statistical approaches estimating variance components, sire models versus animal models, or threshold versus linear models, as well as different prevalence rates in the data as shown by van Hulzen et al. (2011). Koets et al. (2000) classified the positive animals on microbiological and histopathological analyses after slaughtering, whereas most other studies used ELISA to determine specific antibodies against MAP in milk or blood (Mortensen et al., 2004; Gonda et al., 2006; Hinger et al., 2008; van Hulzen et al., 2011). A third factor influencing the heritability estimates could be variances in sample sizes from 684,364 animals (van Hulzen et al., 2011) to 3,020 animals (Koets et al., 2000).

In general, the sensitivity of ELISA tests is low, meaning that infected animals do not always show a positive ELISA test result. Therefore, fecal culture is considered as the most sensitive method for detecting MAP in cattle (Collins et al., 2006) and is deemed the gold standard (Chacon et al., 2004). So far only 1 study on heritability estimates based on fecal culture has been published (Gonda et al., 2006). These estimates are based on a data set from a QTL study with data from 12 sires only, which hardly can be seen as a random sample of sires. The objective of this study was to estimate genetic variation and heritability for paratuberculosis infection in a large data set of German Holstein cows classified as MAP-positive and MAPnegative animals using fecal cultural results.

\section{MATERIALS AND METHODS}

\section{Data Set}

A data set of 11,285 German Holstein herd book cows originating from 15 farms was available. All animals were routinely screened for MAP status in 2008 and 2009 as part of a voluntary program in Thuringia in Germany (Donat, 2009) by fecal culture (AVID, 2007), whereas a total of 1,218 animals $(10.79 \%)$ tested positive and 10,067 MAP negative. For all animals only a single test result was available.

The 11,285 cows descended from a total of 869 sires with an average of 12.8 daughters per sire, but with large differences in the number of daughters among the sires. Three hundred and ninety-two sires had more than 4 daughters, with an average of 25.8 daughters per sire. The maximum was 445 daughters per sire. The distribution of sires and number of daughters is shown in Table 1. For further analyses, the total data set was divided into 2 subsets with data from farms with MAP prevalence rates below $10 \%$ and above $10 \%$. In the first subset $(<10 \%$ prevalence), 9 farms were included with a total of 654 sires and an average number of daughters per sire of 10.3. In the second subset, 6 farms with a total of 468 sires and an average number of daughters per sire of 9.0 were included. The distribution of sires and number of daughters for these 2 subsets is shown in Table 1.

\section{Statistical Analysis}

The statistical analyses (SAS GLIMMIX and ASREML; SAS Institute Inc., Cary, NC) were carried out with logistic regression models using the MAP status as a binomial random variable. The logistic models use the logit link function, which is defined by $\ln \left[\mathrm{p}_{\mathrm{i}} /(1-\right.$ $\left.p_{i}\right)$ ], where $p_{i}$ is the probability of an animal to be MAP positive.

The data were analyzed with the GLIMMIX procedure of SAS 9.1 to estimate the significance of fixed effects on the MAP status of cows. Farm and lactation number as age effect were included in the model as fixed effects. All lactation numbers above 5 were combined into 1 class. To estimate the variance components, farm and lactation number as fixed effects and sire as random effect or, alternatively, animal was included in a threshold model (logit function) using

Table 1. Distribution of number of daughters per sire

\begin{tabular}{lccc}
\hline & \multicolumn{3}{c}{ Number of sires } \\
\cline { 2 - 4 } $\begin{array}{l}\text { Number of } \\
\text { daughters }\end{array}$ & $\begin{array}{c}\text { Complete } \\
\text { data set }\end{array}$ & $\begin{array}{c}\text { Prevalence within } \\
\text { herd }<10 \%\end{array}$ & $\begin{array}{c}\text { Prevalence within } \\
\text { herd }>10 \%\end{array}$ \\
\hline 1 & 206 & 155 & 131 \\
2 & 131 & 109 & 102 \\
3 & 77 & 72 & 47 \\
4 & 63 & 52 & 158 \\
Total & 392 & 266 & 468 \\
\hline
\end{tabular}




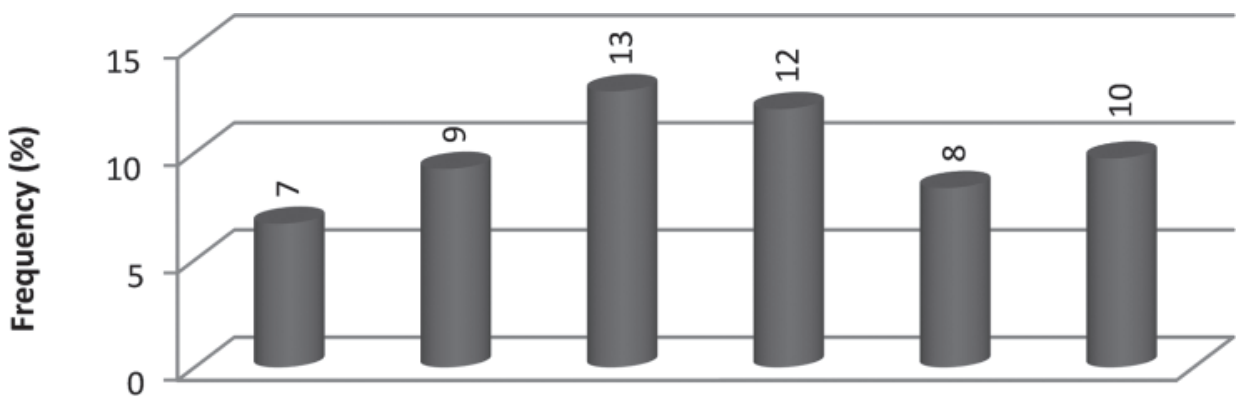

\begin{tabular}{|l|c|c|c|c|c|c|}
\hline Lactation & 1 & 2 & 3 & 4 & 5 & $>6$ \\
\hline Average age (mo) & 31 & 44 & 58 & 71 & 85 & 105 \\
\hline $\mathrm{n}$ & 4,471 & 3,126 & 1,782 & 979 & 450 & 323 \\
\hline
\end{tabular}

Figure 1. Estimated frequency of fecal tested Mycobacterium avium ssp. paratuberculosis (MAP)-positive German Holstein cows per lactation number and average age of the cows per lactation number.

the software package ASREML (Gilmour et al., 2009). Heritabilities were calculated using the logit function, which implies a correction of the residual variance by the factor $\pi^{2} / 3$. Within the sire model, the relationship between sires $(6,535$ animals in total) was included in the model. Within the animal model, the full relationship between all animals (42,195 animals in total) was included in the model. The variance components using the sire model were estimated including all 869 sires and, alternatively, only the 392 sires with more than 4 daughters in the data set.

One farm showed a very high MAP prevalence of $68 \%$, whereas the other farms showed prevalence rates below 30\%. Therefore, the variance components with the sire and the animal models were also estimated excluding the farm with the high prevalence rate. Additionally, the variance components with the sire model and the animal model were also estimated within the 2 subsets of data based on test prevalence: 1) herds with a prevalence $<10 \%$ and 2 ) herds with a prevalence $>10 \%$. The different models were used to analyze the effects of sire versus animal model as well as different herd prevalence rates on heritability estimates for comparison to Hinger et al. (2008) and to van Hulzen et al. (2011).

\section{RESULTS}

The fixed effects farm and number of lactation had a high significant effect $(P<0.001)$ on the MAP status. The estimated MAP frequency based on fecal-positive cows within lactation number and the average age within lactation number are summarized in Figure 1. We observed an increase in the frequency of positive animals from the first to the third lactation. Animals in the third and fourth lactation show the highest values with 13 and $12 \%$, respectively, whereas fecal-positive tested cows with 6 or more lactations showed a frequency of $10 \%$ positive animals. The frequency of MAP-positive animals per farm, shown in Figure 2, varied from 2.8\% (farm number 6) to $67.6 \%$ (farm number 3 ).

The estimated variance components and heritabilities for both models and all 5 data sets are shown in Table 2. Estimated heritabilities range from 7.8 to $28.3 \%$. The highest heritability, as well as the highest additive genetic variance, was estimated in the sire model on herds with prevalence above $10 \%$. The lowest additive genetic variance was estimated with the animal model within herds with prevalence below $10 \%$. The exclusion of farm number 3 did not show an effect on the residual variance for the animal model, but a small decrease in the residual variance for the sire model. The heritability estimates are, therefore, slightly higher than from the complete data set. The sire models showed higher heritabilities than the equivalent animal models for all data sets. For animal and sire models, the data set with prevalence below $10 \%$ showed a much lower heritability than the data set with prevalence above $10 \%$, caused by the much higher estimates of additive genetic variance in both models for the latter data set.

\section{DISCUSSION}

The different prevalence rates within farms are most likely a result of the manner and duration of eradication procedures on the one hand or could be caused by different environmental and management factors on the other. Especially the management of replacement as 


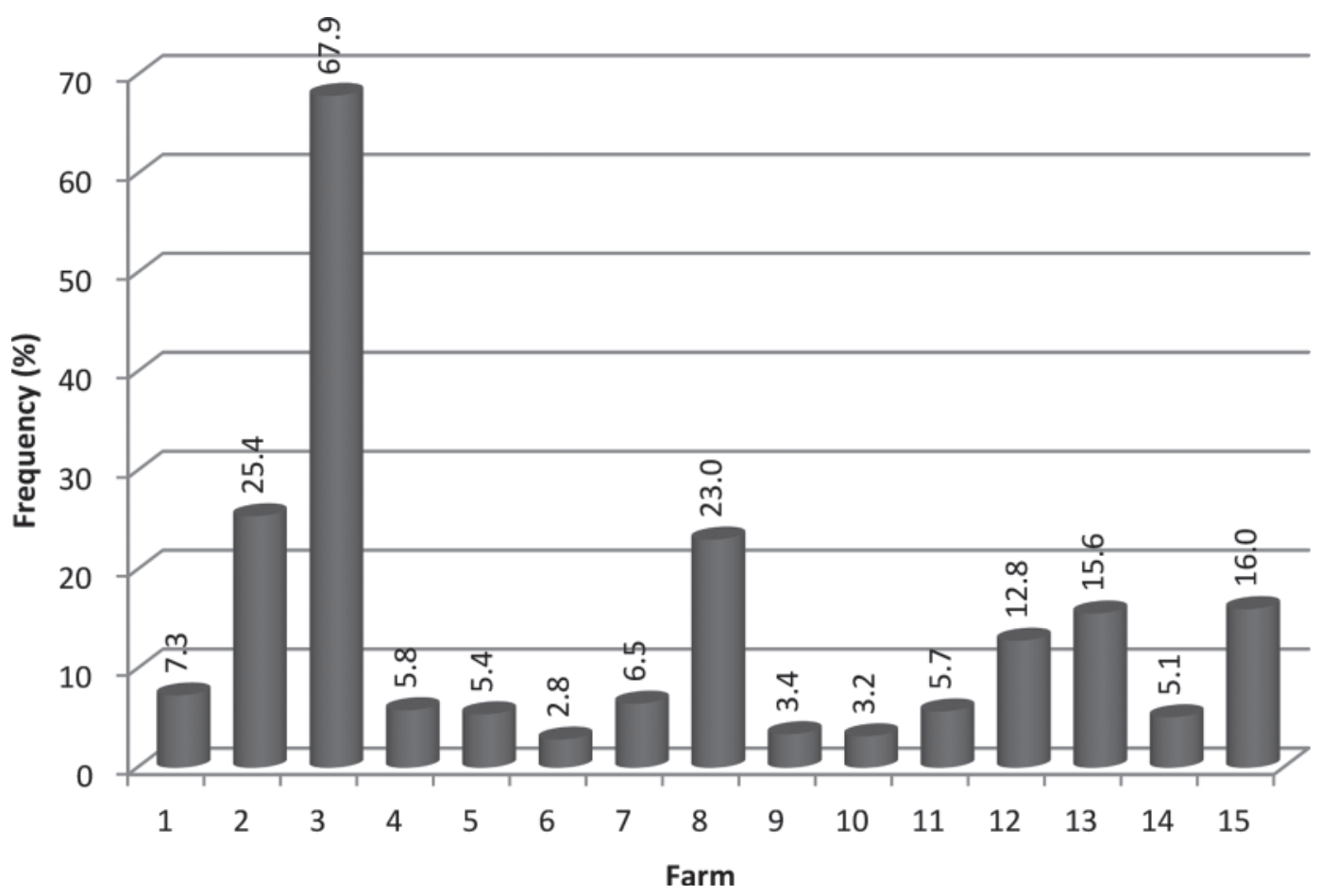

Figure 2. Estimated frequency of fecal-tested Mycobacterium avium ssp. paratuberculosis (MAP)-positive German Holstein cows per farm.

well as the calving and hygiene practices played important roles (Raizman et al., 2004; McKenna et al., 2006; Collins et al., 2010). Farm number 3 with the very high prevalence of almost $68 \%$ had not reacted with culling of positive animals so far. The other farms had already been involved in the voluntary surveillance program in Thuringia for a longer period, but before 2008, blood ELISA test results were used to detect positive and negative animals. The change in prevalence over time within farms has not yet been analyzed and, therefore, a possible selection response cannot be quantified.

Hinger et al. (2008) showed an increase of ELISApositive tested animals up to an age of $5 \mathrm{yr}$. This result corresponds to the increase in positive animals up to the third lactation (see Figure 1) observed in this study with an average age of 58 mo. The present results and those from Jubb and Galvin (2000), Dreier et al. (2006), and Hinger et al. (2008) using ELISA tests leads to the conclusion that both methods (fecal culture and ELISA) show the same tendency of MAP prevalence over age groups. This result was not expected, as Eamens et al. (2000) have shown that fecal culture testing and serology did not show a high correlation. They confirmed only $37 \%$ of fecal positive cattle by ELISA testing. For a subgroup of animals from the total data set in this study, both fecal culture as well as ELISA test results are available (publishing in progress). Due to its higher sensitivity, as shown by McKenna et al. (2005) and Köhler et al. (2008), testing by fecal culture results in a higher prevalence then the ELISA test

Table 2. Estimates of heritabilities $\left(\mathrm{h}^{2}\right)$ with standard errors and additive genetic $\left(\mathrm{V}_{\mathrm{A}}\right)$ and phenotypic variances $\left(V_{P}\right)$ for different models and data sets

\begin{tabular}{lclccc}
\hline Data set & $\mathrm{n}$ & Model & $\mathrm{h}^{2} \pm \mathrm{SE}$ & $\mathrm{V}_{\mathrm{A}}$ & $\mathrm{V}_{\mathrm{P}}$ \\
\hline 1: Complete & \multirow{2}{*}{11,129} & Sire & $0.195 \pm 0.054$ & 0.675 & 3.469 \\
& & Animal & $0.157 \pm 0.024$ & 0.613 & 3.913 \\
2: Sires with $>$ 4 daughters & 10,126 & Sire & $0.175 \pm 0.051$ & 0.602 & 3.451 \\
3: Without farm number 3 & 10,555 & Sire & $0.228 \pm 0.061$ & 0.799 & 3.500 \\
& & Animal & $0.168 \pm 0.026$ & 0.666 & 3.966 \\
4: Prevalence within herd $<10 \%$ & \multirow{2}{*}{6,823} & Sire & $0.112 \pm 0.091$ & 0.378 & 3.395 \\
& \multirow{2}{*}{4,306} & Animal & $0.078 \pm 0.051$ & 0.278 & 3.578 \\
5: Prevalence within herd $>10 \%$ & & Sire & $0.283 \pm 0.078$ & 1.006 & 3.551 \\
& & Animal & $0.138 \pm 0.028$ & 0.771 & 4.071 \\
\hline
\end{tabular}


results. Only a very small proportion of fecal-negative tested animals were positive using ELISA tests $(<5 \%)$, which is a consequence of the limited specificity (McKenna et al., 2005; Köhler et al., 2008) of the ELISA tests. From the development shown above, it could be assumed that infected animals detected by ELISA tests react simultaneously positive in fecal culture.

The estimated heritabilities from the complete data set of the binary criteria fecal positive or negative between $15.7 \%$ for the animal and $19.5 \%$ for the sire model are in agreement with Gonda et al. (2006). Based on fecal-tested animals, they estimated heritability in US Holsteins to be $15.3 \%$. The heritability based on ELISA tests are between 0.031 and 0.16, depending on ELISA test (milk or blood) and the breed (Mortensen et al., 2004; Gonda et al., 2006; Hinger et al., 2008; Attalla et al., 2010; van Hulzen et al., 2011).

In our study, the estimated heritability using the animal model was lower than the estimated heritability by using the sire model for all data sets, which is in agreement with Hinger et al. (2008). In cases of sires with a small number of daughters, an advantage exists in using an animal model rather than a sire model (Ramirez-Valverde et al., 2001). These results do not support the assumption made by van Hulzen et al. (2011) that an animal model will estimate higher additive genetic variance due to possible intrauterine infections of calves with MAP. Comparing the results from the sire model and the sire model with more than 4 daughters (Table 2) confirmed the results of RamirezValverde et al. (2001). The latter model shows a lower heritability than the other sire model, caused by the lower estimated genetic variance, which is close to the estimated genetic variance of the animal model from the same data set. Farm number 3 with the high prevalence did not affect the phenotypic variance but slightly increased the additive genetic variance estimate. This is not in agreement with the effect of higher heritability estimates from data with increasing within-herd prevalence as found by van Hulzen et al. (2011) and also confirmed in the current study from the 2 subsets of data based on prevalence within farms. The sire and the animal models from the subset with prevalence within herds below 10\% show a much lower additive genetic variance than the data set with prevalence within herds above $10 \%$. This effect could be explained on one hand by a possible selection effect through culling of positive-tested animals. On the other hand, it is well known that the additive genetic variance in the case of a trait influenced by only a single gene with 2 alleles is dependent on the allele frequency, and with decreasing allele frequency, additive genetic variance also decreases. In case the MAP status is affected by only a small number of genes, the decrease in additive genetic variance is expected with decreasing prevalence within herds and also with increasing prevalence above $50 \%$, as farm number 3 shows.

In the case of breeding, it seems advantageous to use fecal diagnostics instead of ELISA tests to detect positive animals because of the higher heritabilities found. Taking into consideration the low conformance between fecal and ELISA test results as shown by Eamens et al. (2000), open questions still exist about culling of animals to achieve MAP-free status success. Also, with respect to future genomic selection, it is necessary to improve the diagnostics for MAP status to achieve high conformance between different test methods.

\section{CONCLUSIONS}

The heritability for the susceptibility to MAP estimates of fecal culture results are between 7.8 and $28.3 \%$ from sire and animal models and different data sets. The results of the study confirm a low to medium genetic background for susceptibility to paratuberculosis infection in dairy cows. The heritability indicates that fecal culture diagnosis is a useful tool as a selection criterion against Johne's disease to increase resistance in cattle. Moreover, it is useful as a selection criterion in breeding programs as well as for further studies on genomic selection or candidate genes.

\section{ACKNOWLEDGMENTS}

We are grateful to the herd book breeders from Thuringia, Germany, for participating in the voluntary program and for providing the data.

\section{REFERENCES}

Attalla, S. A., A. J. Seykora, J. B. Cole, and B. J. Heins. 2010. Genetic parameters of milk ELISA scores. J. Dairy Sci. 93:1729-1735.

AVID (Arbeitskreis Veterinärmedizinische Infektionsdiagnostik). 2007. Kultureller Nachweis von Mycobacterium avium ssp. paratuberculosis in Kot- oder Organproben. Deutsche Veterinärmedizinische Gesellschaft (DVG) e. V, Gießen, Germany.

Böttcher, J., and A. Gangl. 2004. Mycobacterium avium ssp. Paratuberculosis - Combined serological testing and classification of individual animals and herds. J. Vet. Med. B Infect. Dis. Vet. Public Health 51:443-448.

Chacon, O., L. E. Bermudez, and R. G. Barletta. 2004. Johne's disease, inflammatory bowel disease, and Mycobacterium paratuberculosis. Annu. Rev. Microbiol. 58:329-363.

Chiodini, R. J., H. J. Van Kruiningen, and R. S. Merkal. 1984. Ruminant paratuberculosis (Johne's disease): The current status and future prospects. Cornell Vet. 74:218-262.

Collins, M. T., V. Eggleston, and E. J. B. Manning. 2010. Successful control of Johne's disease in nine dairy herds: Results of a six-year field trial. J. Dairy Sci. 93:1638-1643.

Collins, M. T., I. A. Gardner, F. B. Garry, A. J. Roussel, and S. J. Wells. 2006. Consensus recommendations on diagnostic testing for the detection of paratuberculosis in cattle in the United States. J. Am. Vet. Med. Assoc. 229:1912-1919. 
Donat, K. 2009. Umsetzung des Thüringer Paratuberkulose-Landesprogrammes auf der Basis des Erregernachweises - Ein Zwischenbericht. Page 94 in Zusammenfassung 7. Stendaler Symposium. Landesamt für Verbraucherschutz Sachsen-Anhalt, Fachbereich 4 Veterinärmedizin, Stendal, Germany.

Donat, K., U. Schau, and A. Soschinka. 2011. Identification of Mycobacterium avium ssp. paratuberculosis infected dairy herds by environmental sampling. Berl. Münch. Tierärztl. Wochenschr. 124:360-367.

Dreier, S., J. L. Khol, B. Stein, K. Fuchs, S. Gütler, and W. Baumgartner. 2006. Serological, bacteriological and molecularbiological survey of paratuberculosis (Johne's disease) in Austrian cattle. J. Vet. Med. B Infect. Dis. Vet. Public Health 53:477-481.

Eamens, G. J., R. J. Whittington, I. B. Marsh, M. J. Turner, V. Saunders, P. D. Kemsley, and D. Rayward. 2000. Comparative sensitivity of various faecal culture methods and ELISA in dairy cattle herds with endemic Johne's disease. Vet. Microbiol. 77:357-367.

Gasteiner, J., H. Wenzl, K. Fuchs, U. Jark, and W. Baumgartner. 1999. Serological cross-sectional study of paratuberculosis in cattle in Austria. Zentralbl. Veterinarmed. B 46:457-466.

Gilmour, A. R., B. J. Gogel, B. R. Cullis, and R. Thompson. 2009. ASReml User Guide Release 3.0. VSN International Ltd., Hemel Hempstead, UK.

Gonda, M. G., Y. M. Chang, G. E. Shook, M. T. Collins, and B. W. Kirkpatrick. 2006. Genetic variation of Mycobacterium avium ssp. paratuberculosis infection in US Holsteins. J. Dairy Sci. 89:18041812.

Harris, N. B., and R. G. Barletta. 2001. Mycobacterium avium ssp. paratuberculosis in veterinary medicine. Clin. Microbiol. Rev. 14:489-512.

Hendrick, S., T. Duffield, K. Leslie, K. Lissemore, M. Archambault, and D. Kelton. 2005. The prevalence of milk and serum antibodies to Mycobacterium avium subspecies paratuberculosis in dairy herds in Ontario. Can. Vet. J. 46:1126-1129.

Hinger, M., H. Brandt, and G. Erhardt. 2008. Heritability estimates for antibody response to Mycobacterium avium subspecies paratuberculosis in German Holstein cattle. J. Dairy Sci. 91:3237-3244.

Jubb, T., and J. Galvin. 2000. Herd testing to control bovine Johne's disease. Vet. Microbiol. 77:423-428.

Koets, A. P., G. Adugna, L. L. G. Janss, H. J. van Weering, C. H. J. Kalis, G. H. Wentink, V. P. M. G. Rutten, and Y. H. Schukken. 2000. Genetic variation of susceptibility to Mycobacterium avium ssp. paratuberculosis infection in dairy cattle. J. Dairy Sci. 83:2702-2708.
Köhler, H., B. Burkert, I. Pavlik, R. Diller, L. Geue, F. J. Conraths, and G. Martin. 2008. Evaluation of five ELISA test kits for the measurement of antibodies against Mycobacterium avium subspecies paratuberculosis in bovine serum. Berl. Münch. Tierärztl. Wochenschr. 121:203-210.

McKenna, S. L. B., G. P. Keefe, H. W. Barkema, and D. C. Sockett. 2005. Evaluation of three ELISAs for Mycobacterium avium ssp. paratuberculosis using tissue and fecal culture as comparison standards. Vet. Microbiol. 110:105-111.

McKenna, S. L. B., G. P. Keefe, A. Tiwari, J. VanLeeuwen, and H. W. Barkema. 2006. Review: Johne's disease in Canada part II: Disease impacts, risk factors, and control programs for dairy producers. Can. Vet. J. 47:1089-1099.

Mortensen, H., S. S. Nielsen, and P. Berg. 2004. Genetic variation and heritability of the antibody response to Mycobacterium avium subspecies paratuberculosis in Danish Holsteins cows. J. Dairy Sci. 87:2108-2113.

Muskens, J., H. W. Barkema, E. Russchen, K. van Maanen, Y. H. Schukken, and D. Bakker. 2000. Prevalence and regional distribution of paratuberculosis in dairy herds in the Netherlands. Vet. Microbiol. 77:253-261.

Nielsen, S. S., and N. Toft. 2008. Ante mortem diagnosis of paratuberculosis: A review of accuracies of ELISA, interferon- $\gamma$ assay and faecal culture techniques. Vet. Microbiol. 129:217-235.

Raizman, E. A., S. J. Wells, S. M. Godden, R. F. Bey, M. J. Oakes, D. C. Bentley, and K. E. Olsen. 2004. The distribution of Mycobacterium avium ssp. paratuberculosis in the environment surrounding Minnesota dairy farms. J. Dairy Sci. 87:2959-2966.

Ramirez-Valverde, R., I. Misztal, and J. K. Bertrand. 2001. Comparison of the threshold vs. linear and animal vs. sire models for predicting direct and maternal genetic effects on calving difficulty in beef cattle. J. Anim. Sci. 79:333-338.

van Hulzen, K. J. E., M. Nielen, A. P. Koets, G. de Jong, J. A. M. van Arendonk, and H. C. M. Heuven. 2011. Effect of herd prevalence on heritability estimates of antibody response to Mycobacterium avium subspecies paratuberculosis. J. Dairy Sci. 94:992-997.

Wells, S. J., R. H. Whitlock, B. A. Wagner, J. Collins, F. Garry, H. Hirst, J. Lawrence, W. J. Saville, and A. L. L. Naugle. 2002. Sensitivity of test strategies used in the Voluntary Johne's Disease Herd Status Program for detection of Mycobacterium paratuberculosis infection in dairy cattle herds. J. Am. Vet. Med. Assoc. 220:1053-1057. 